\title{
Rethinking Resection and Transplant Candidacy for HCC: Should Tumor Biology Replace Size-Based Criteria?
}

\author{
Alexandra W. Acher, MD, and Daniel E. Abbott, MD \\ Department of Surgery, University of Wisconsin School of Medicine and Public Health, Madison
}

The current issue of Annals of Surgical Oncology includes a study by Yan and colleagues titled "Integrated nomograms for the preoperative prediction of microvascular invasion and lymph node metastasis risk in hepatocellular carcinoma patients." 1 This study describes a nomogram, constructed from various preoperative variables, that predicts tumor microvascular invasion (MVI) with a sensitivity of $81.5 \%$ and specificity of $75.3 \%$ in training and validation patient cohorts (C-score of 0.828 and 0.804 respectively). Although this is not the first or largest nomogram-based study developed to predict MVI from preoperative factors, it has a comparatively high sensitivity and specificity and is part of a larger conversation regarding appropriate patient selection for liver resection and transplant in patients with hepatocellular carcinoma (HCC). ${ }^{1,2}$ The nomogram also includes a predictive nomogram for lymph node positivity, which has clinical value in risk-stratifying patients before major surgical intervention. However, the sensitivity and specificity of the nomogram for node positivity preclude it from being used as an active clinical tool and thus discussion of the clinical implications of their MVI prediction nomogram will be more instructive.

In surgical candidates, curative intent liver resection and liver transplant are the most effective HCC treatments. ${ }^{3}$ Unfortunately, recurrence after transplant is $30 \%$ at 12 months and $50-75 \%$ at 5 years, whereas recurrence after liver resection is $50 \%$ at 2 years and $75 \%$ at 5 years. $^{3-7}$ While there are many risk factors for

(C) Society of Surgical Oncology 2019

First Received: 30 October 2019;

Published Online: 14 November 2019

D. E. Abbott, MD

e-mail: abbott@surgery.wisc.edu recurrence, MVI is the risk factor with the strongest association with early and late recurrence. ${ }^{5-8,12,13}$ MVI also is associated with decreased disease-free and overall survival. $^{8,9}$ Although MVI is associated with increasing tumor size, it exists in small tumors and is absent in some larger tumors; in retrospective studies, MVI was present in $20 \%$ of tumors $<2 \mathrm{~cm}, 30-60 \%$ of tumors between $2-5 \mathrm{~cm}$, and $60-90 \%$ of tumors $>5 \mathrm{~cm} .^{6,10}$ Because MVI is strongly associated with recurrence and an independent marker of aggressive tumor biology, some have advocated that liver transplant selection criteria rely on tumor biology-related factors rather than tumor number and size-based criteria. $^{7,10,11}$

The use of MVI as a surgical decision making tool, however, is dependent on the ability to confidently and consistently identify MVI preoperatively. Although considerable effort has been devoted to developing MVI prediction scores, the data are varied and no definitive prospective study has validated a clinically useful predictive score. Lei et al. developed a nomogram to predict MVI based on preoperative patient and tumor factors with a training group of 707 patients and a validation group of 297 patients (C-score 0.81 and 0.80 respectively). Their MVI prediction nomogram includes increasing tumor diameter $(<1$ to $>5 \mathrm{~cm})$, increasing serum AFP $(<20,20-400$, $>400)$, HBV DNA $>10^{4}$, platelets $<100$, typical dynamic on contrast-enhanced MRI (i.e., arterial phase hyper-attenuation and portal venous and delayed phase hypo-attenuation), multiple tumors, and incomplete encapsulation. ${ }^{1}$ Their nomogram achieved a sensitivity of $73.5 \%$ and a specificity of $77 \%$.

Other groups have published MVI prediction nomograms based on inflammatory biomarkers with the hypothesis that inflammatory processes are associated with more aggressive tumor biology. With 627 patients, a nomogram using neutrophil-to-lymphocyte ratio, direct 
neutrophil to lymphocyte ratio, and tumor volume nomogram achieved a sensitivity of $51 \%$ and a specificity of $81 \%$ (C-score 0.727 training and 0.839 validation). ${ }^{2}$

Additionally, imaging-defined risk factors for recurrence and MVI have been investigated. Although CT and MRI are both used to diagnose HCC, hepatocyte specific contrast (gadoxetic acid) has greatly improved the sensitivity and specificity of MRI over that of CT and can demonstrate microvascular anatomy, including invasion. ${ }^{12-14}$ That said, even with these advances, MVI remains difficult to reliably predict. $^{12}$

Several aspects of the current study by Yan and colleagues distinguish it from existing studies. One explicit goal was to create a nomogram that relied on easily attainable and inexpensive diagnostics. This priority is reflected in the components of the blood index: AFP, CA125, CA 19-9, gamma-glutamyl transferase (GGT), aspartate aminotransferase (AST), albumin, white blood cells, platelets, alkaline phosphatase (ALP). While employing daily labs in a clinical score increases the ease of using this tool, it also prompts discussion of the clinical reasoning and methodology of selection criteria. For instance, thrombocytopenia has been included in other predictive scores as a measure of MVI related to splenic platelet sequestration secondary to sinusoidal congestion that defines MVI and is a sequelae of cirrhosis. ${ }^{1,15}$ However, the present study suggests platelets $>150$ are associated with an increased risk of MVI as denoted by its positively weighted blood index coefficient. Additionally, CA-125 and CA 19-9 are included in the blood index. While CA125 and CA 19-9 have been examined as adjuncts to AFP in the diagnosis of HCC, they have variable sensitivity and specificity for detection of $\mathrm{HCC}$, with unclear clinical implication, because they also are elevated in cholestasis and therefore are not routinely used in most U.S. cancer centers. ${ }^{16-18}$ The author's methodology for identifying the components of their blood index relies on algorithmic processing to select optimal combinations of variables. While this process identifies the most predictive combination of variables from a larger group within this patient cohort, the small study size as well as the unclear clinical reasoning for some variable inclusions make it difficult to interpret. Although likely more expensive and resource dependent, algorithmic identification of the optimal combination of HCC tumor markers, such as Dex-ycarboxyprothrombin (DCP), vascular endothelial growth factor (VEGF), GGT, and Glypican-3 (GPC3) may be an interesting strategy to quantify aggressive tumor biology. ${ }^{19}$

The authors' analysis of the tumor number on MVI risk needs clinical justification. They compute a greater risk of MVI for 2 nodules [odds ratio (OR) 2.6] than for multiple nodules (OR 1.2) or a solitary nodule (OR 1), but their nomogram score weights a solitary nodule (score of 13 ) with a higher risk for MVI than 2 nodules (score of 7) or multiple nodules (score of 0 ). This contradiction is confusing, and is in contrast to other reports that indicate increasing tumor number as a risk factor for MVI. ${ }^{1}$ Additionally, this information contradicts their nomogram scoring of BCLC stage where increasing BCLC stage has a higher risk of MVI; this is especially evident for BCLC Stage 0-B where tumor number plays a distinguishing role compared with other BCLC stage components (performance status and degree of cirrhosis). ${ }^{20}$

The authors also relied on CT as their diagnostic and staging imaging modality. Although this may reflect regional standard of care, MR is superior to $\mathrm{CT}$ in the detection and characterization of HCC (tumor size, number, and encapsulation), especially in a background of cirrhosis and for tumors $<2 \mathrm{~cm} .{ }^{13,14,21}$ Because the goal is to develop the most accurate nomogram possible, and one that is generalizable to centers that routinely use MRI, one would ideally utilize MRI (rather than CT) to stage HCC, because it has the highest potential for accuracy. This is especially important when considering small tumors and multiple tumors in predictive scores, as the sensitivity of contrast enhanced MRI to diagnose $\mathrm{HCC}<2 \mathrm{~cm}$ is $90-95 \%$, whereas that of CT diminishes to $<60 \% .{ }^{14,21}$

Disease prevalence also impacts the positive predictive value of nomograms: a predictive score with a given sensitivity will have a better positive predictive value in a population with high disease prevalence than in a population with low disease prevalence. ${ }^{22}$ Given the high HCC prevalence in Asia and Africa compared with Europe and North America, this becomes very relevant for the wider applicability of predictive scores. Additionally, region specific differences in $\mathrm{HCC}$ etiology will potentially impact the variables included in predictive scores of disease and disease features. ${ }^{23}$

The current study by Yan and colleagues is an important contribution to a larger conversation regarding how tumor specific factors may influence surgical candidacy in HCC. As our understanding of markers of tumor biology and predictors of aggressive disease increases, quantification of tumor biology may replace size-based criteria for resection and transplant candidates. However, until there are predictive scores created from clinically rational methodologies that consistently achieve high predictive accuracy, it is difficult to study the impacts of altering patient selection criteria for resection or transplant.

ACKNOWLEDGEMENTS Dr. Acher is supported by a T32 grant from the National Cancer Institute associated with the Division of Surgical Oncology at the University of Wisconsin School of Medicine and Public Health (UWSMPH). The UWSMPH Surgical Oncology T32 Research Fellowship offers 2 years of formal training in the design, organization, conduct, analyses, and publication of surgical 
oncology research with an emphasis on understanding the complexities of translating science into clinical practice.

DISCLOSURES The authors declare no conflicts of interest.

\section{REFERENCES}

1. Yan $\mathrm{Y}$, Zhou $\mathrm{Q}$, Zhang $\mathrm{M}$, et al. Integrated nomograms for the preoperative prediction of microvascular invasion and lymph node metastasis risk in hepatocellular carcinoma patients. Ann Surg Oncol. 2019. https://doi.org/10.1245/s10434-019-0807 1-7.

2. Lei Z, Li J, Wu D, Xia Y, Wang Q, Si A, et al. Nomogram for preoperative estimation of microvascular invasion risk in hepatitis $\mathrm{b}$ virus-related hepatocellular carcinoma within the Milan Criteria. JAMA Surg. 2016;151(4):356-63.

3. Li P, Huang W, Wang F, Ke YF, Gao L, Shi KQ, et al. Nomograms based on inflammatory biomarkers for predicting tumor grade and micro-vascular invasion in stage I/II hepatocellular carcinoma. Biosci Rep. 2018. https://doi.org/10.1042/ BSR20180464.

4. Daher S, Massarwa M, Benson AA, Khoury T. Current and future treatment of hepatocellular carcinoma: an updated comprehensive review. J Clin Transl Hepatol. 2018;6(1):69-78.

5. Rahimi RS, Trotter JF. Liver transplantation for hepatocellular carcinoma: outcomes and treatment options for recurrence. Ann Gastroenterol. 2015;28(3):323-30.

6. Cescon M, Ravaioli M, Grazi GL, Ercolani G, Cucchetti A, Bertuzzo V, et al. Prognostic factors for tumor recurrence after a 12-year, single-center experience of liver transplantations in patients with hepatocellular carcinoma. $J$ Transplant. 2010. http s://doi.org/10.1155/2010/904152.

7. Llovet JM, Schwartz M, Mazzaferro V. Resection and liver transplantation for hepatocellular carcinoma. Semin Liver Dis. 2005;25(2):181-200.

8. Kornberg A. Liver transplantation for hepatocellular carcinoma beyond Milan Criteria: multidisciplinary approach to improve outcome. ISRN Hepatol. 2014;2014:706945.

9. Roayaie S, Blume IN, Thung SN, Guido M, Fiel MI, Hiotis S, et al. A system of classifying microvascular invasion to predict outcome after resection in patients with hepatocellular carcinoma. Gastroenterology. 2009;137(3):850-5.

10. Zhang X, Li J, Shen F, Lau WY. Significance of presence of microvascular invasion in specimens obtained after surgical treatment of hepatocellular carcinoma. J Gastroenterol Hepatol. 2018;33(2):347-54.

11. Pawlik TM, Delman KA, Vauthey JN, Nagorney DM, Ng IO, Ikai I, et al. Tumor size predicts vascular invasion and histologic grade: Implications for selection of surgical treatment for hepatocellular carcinoma. Liver Transpl. 2005;11(9):1086-92.
12. Vauthey JN, Lauwers GY, Esnaola NF, Do KA, Belghiti J, Mirza $\mathrm{N}$, et al. Simplified staging for hepatocellular carcinoma. J Clin Oncol. 2002;20(6):1527-36.

13. Cuccurullo V, Di Stasio GD, Mazzarella G, Cascini GL.. Microvascular invasion in HCC: the molecular imaging perspective. Contrast Media Mol Imaging. 2018;2018:9487938.

14. Hammerstingl R, Huppertz A, Breuer J, Balzer T, Blakeborough A, Carter R, et al. Diagnostic efficacy of gadoxetic acid (Primovist)-enhanced MRI and spiral CT for a therapeutic strategy: comparison with intraoperative and histopathologic findings in focal liver lesions. Eur Radiol. 2008;18(3):457-67.

15. Raman SS, Leary C, Bluemke DA, Amendola M, Sahani D, McTavish JD, et al. Improved characterization of focal liver lesions with liver-specific gadoxetic acid disodium-enhanced magnetic resonance imaging: a multicenter phase 3 clinical trial. J Comput Assist Tomogr. 2010;34(2):163-72.

16. Rungsakulkij N, Mingphruedhi S, Suragul W, Tangtawee P, Muangkaew P, Aeesoa S.. Platelet-to-lymphocyte ratio and large tumor size predict microvascular invasion after resection for hepatocellular carcinoma. Asian Pac $J$ Cancer Prev. 2018;19(12):3435-41.

17. Fabris C, Basso DA, Leandro G, Meggiato T, Elba S, Panozzo MP, et al. Serum CA 19-9 and alpha-fetoprotein levels in primary hepatocellular carcinoma and liver cirrhosis. Cancer. 1991;68(8):1795-8.

18. Hsu CC, Goyal A, Iuga A, Krishnamoorthy S, Lee V, Verna EC, et al. Elevated CA19-9 is associated with increased mortality in a prospective cohort of hepatocellular carcinoma patients. Clin Transl Gastroenterol. 2015;6:e74.

19. Giannini E, Borro P, Botta F, Chiarbonello B, Fasoli A, Malfatti F, et al. Cholestasis is the main determinant of abnormal CA 19-9 levels in patients with liver cirrhosis. Int $\mathrm{J}$ Biol Markers. 2000;15(3):226-30.

20. Zhao YJ, Ju Q, Li GC. Tumor markers for hepatocellular carcinoma. Mol Clin Oncol. 2013;1(4):593-8.

21. Pons F, Varela M, Llovet JM. Staging systems in hepatocellular carcinoma. HPB (Oxford). 2005;7(1):35-41.

22. Hwang J, Kim SH, Lee MW, Lee JY. Small $(\leq 2 \mathrm{~cm})$ hepatocellular carcinoma in patients with chronic liver disease: comparison of gadoxetic acid-enhanced 3.0 T MRI and multiphasic 64-multirow detector CT. $\mathrm{Br} J$ Radiol. 2012;85(1015):e314-22.

23. Brenner H, Gefeller O. Variation of sensitivity, specificity, likelihood ratios and predictive values with disease prevalence. Stat Med. 1997;16(9):981-91.

Publisher's Note Springer Nature remains neutral with regard to jurisdictional claims in published maps and institutional affiliations. 